\title{
Solar cycle 23 analysis
}

\author{
Georgeta Mariş ${ }^{1}$, Miruna Daniela Popescu ${ }^{1,2}$ \\ and Diana Beşliu ${ }^{1}$ \\ ${ }^{1}$ Astronomical Institute of the Romanian Academy Str. Cutitul de Argint 5, RO-040558 1 \\ Bucharest, Romania e-mail: gmaris@aira.astro.ro \\ ${ }^{2}$ Armagh Observatory, College Hill, Armagh, BT61 9DG, N. Ireland email: mdp@arm.ac.uk
}

\begin{abstract}
Here we analyse the solar cycle (SC) 23 behavior and we also make a comparison with some previous cycles and present a few aspects concerning the forecasts made for SC 23 maximum. As regards the following cycle, in accordance to other early predictions, our empirical method, based on observing the flare energy release during the descendant phase of the precedent SC, estimate that the amplitude of SC 24 will be low.
\end{abstract}

\section{Previous forecasts and a short review of SC 23}

A large range of forecasting methods is used to predict the occurrence and amplitude of SCs. They have changed from simple statistical (linear/nonlinear) to complex physical (precursor), based on the understanding of the dynamo process that generates the solar magnetic field and its evolution (Joselyn et al. (1997); Sello (2003); Schatten et al. (2003)).

According to the smoothed sunspot relative number (SSR no.), SC 23 began in May 1996. Based on many prediction methods, it was originally expected to reach a magnitude comparable to SC $21\left(S S R_{\max }^{21}=164.5\right)$ and even higher. A summary of forecasts for its maximum is given by Mariş et al. (2003). $S S R_{\max }^{23}$ was expected to be between 140 and 200, with the exception of a climatologycal method, which had an estimation of 115. SC 23 maximum level was $S S R_{\max }^{23}=120.8$, reached in Apr. 2000, with a second (smaller) maximum in Nov. 2001, of 115.5.

The SSR no. for SCs 20 to 23 is given in Fig. 1 a. For SC 23 current data are only for 90 months from its minimum (May 1996 - Dec. 2003). The SSR of SC 23 is lower than for SCs 21 and 22 and closer to SC 20. Previous SCs with similar $S S R_{\max }$ (see Fig. $1 b$ ) had lengths between $9 \mathrm{yr}$ and 1 month (SC 2) and $11 \mathrm{yr}$ and 10 months (SC 11). SC 23 seems to be much alike SC 17, which had a length of $10 \mathrm{yr}$ and 6 months.

The first SC 23 active region (AR) emerged in May 1996 - late, in comparison with previous SCs, therefore it might give a hint on the cycle's slow amplification and low activity level. SC 23 ARs became predominant over the ones of SC 22 after Feb. 1997. Its ascending phase starts to develop in Sept. 1997. SC 23 had a series of long duration ARs, efficient in high energy eruptive events. They took place in: Jul. 2000, Mar. - Apr. 2001, May - Jun. 2002 and Oct. - Nov. 2003 (Mariş et al., 2002).

We think that by looking at those pulses on the descending phase, which are more obvious in flare energy release indices than in SSR, we can have information about the activity in the current cycle, and even about the appearance of the following one. Those ARs might appear because of the interaction between the magnetic fields, belonging to the old and to the new solar cycle, with a reversed dipole. This behavior of flares could explain the "abnormal" and unpredicted appearance of SC 23. The new magnetic dipole, responsible for SC 23 activity, began to lose part of its energy even during the descending phase of SC 22, so that the activity of SC 23 proves to be well below predicted values. 
(a)

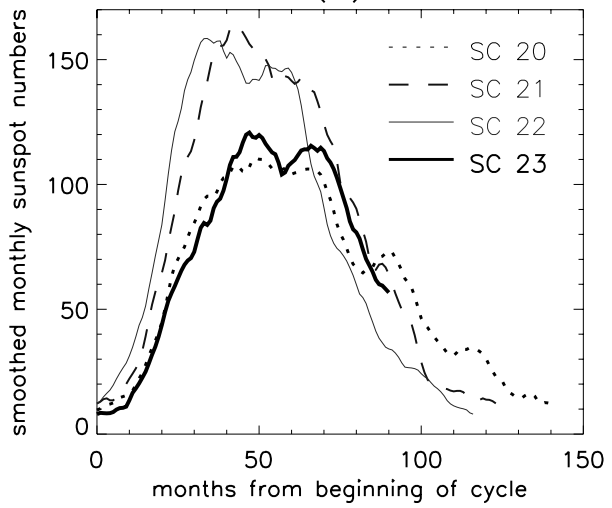

(b)

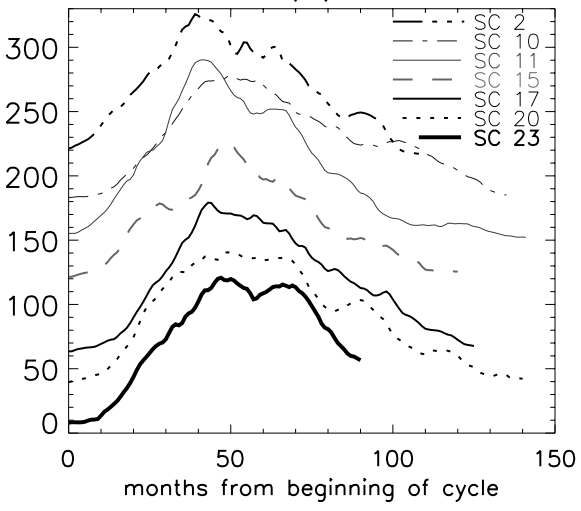

Figure 1. (a) the SSR no. for SCs 20 to 23 . For SC 23 current data are only for 90 months from its minimum (May 1996 to Dec. 2003); (b) previous SCs with similar maximum values for SSR as compared to SC $23\left(S S R_{\max }^{23}=120.8\right)$ : SC 2: 9 yr and 1 month, $S S R_{\max }^{2}=115.8$; SC 10: 11 yr and 4 months, $S S R_{\max }^{10}=98.0$; SC 11: $11 \mathrm{yr}$ and 10 months, $S S R_{\max }^{11}=140.3$; SC $15: 10 \mathrm{yr}$ and 1 month, $S S R_{\max }^{15}=105.4$; SC 17: $10 \mathrm{yr}$ and 6 months, $S S R_{\max }^{17}=119.2$; SC $20: 11 \mathrm{yr}$ and 9 months, $S S R_{\max }^{20}=110.6$. In (b) each cycle was lifted with 40 units for clarity.

\section{Some early forecasts for SC 24}

Preliminary estimations for the following SC support a tendency towards a reduction in the intensity of solar activity. Using a solar dynamo precursor method, Schatten and Tobiska (2003) predict a rapid decline, starting with SC 24. They estimate that the Sun may be heading towards a "Maunder" type of minimum - an extensive period of reduced levels for activity. Sello (2003) uses both a nonlinear and a precursor method, giving a peak amplitude as $S S R_{\max }^{24}=96 \pm 25$, occurring in Apr. 2011 and as $115 \pm 21$ for 2011, respectively. Another early nonlinear prediction $\left(S S R_{\max }^{24}=87 \pm 23.5\right)$ was given by Duhau (2003), who also presents evidence that solar activity is in a decline that started in 1993.

According to our empirical method of estimating the solar activity, based on observing the flare energy release during the descendant phase of the precedent SC, the recent events from Oct. - Nov. 2003 and the likely existence of some upcoming similar ones make us agree that the amplitude of SC 24 will be comparable to SC 23 or even lower.

\section{Acknowledgements}

G. M. would like to acknowledge the receivement of an IAU travel grant.

\section{References}

Duhau, S. 2003 Solar Phys. 213, 20.

Durney, B. R. 2000 Solar Phys. 196, 421.

Joselyn, J. A., Anderson, J. B., Coffey, H., Harvey, K., Hathaway, D., Heckman, G., Hildner, E., Mende, W., Schatten, K., Thompson, R., Thompson, A. W. P., White, O. 1997 EOS, Trans. Amer. Geophys. Union 78, 205.

Mariş, G., Popescu, M. D., Beşliu, D. 2003 Rom. Astron. J. 13, 139.

Mariş, G., Popescu, M. D., Mierla, M. 2002 Rom. Astron. J. 12, 131.

Popescu, M. D., Mariş, G., Oncica, A., Mierla, M. 2002 ESA SP-506, 721.

Sello, S. 2003 Astron. Astrophys. 410, 691.

Schatten, K. H. \& Tobiska, W. K. 2003 American Astronomical Society, SPD meeting 34.

Vitinskii, Yu. I. 1965 NASA TT F-289, NASA, Washington, D. C. 129. 